\title{
Equivalent Capacity-Based Joint Multilevel Coding and Space-Time Transmit Diversity Design
}

\author{
Ronald Yee Siong Tee, Osamah Rashed Alamri, Soon Xin Ng, and Lajos Hanzo, Fellow, IEEE
}

\begin{abstract}
A novel space-time block-coding-assisted and sphere-packed multilevel-coded (STBC-SP-MLC) modulation arrangement is proposed, which combines an MLC-based scheme with a twin-antenna-aided STBC for the sake of providing a transmit diversity gain. The SP modulation invokes a 4-D $D_{4}$ constellation space. A new bit-to-SP symbol mapper is designed for the sake of improving the system's performance. This involves mapping the MLC bits to the SP symbols of Layer-1 and Layer-2 in $D_{4}$ space. The Binary Switching Algorithm is used to determine the optimum cost function used for designing different mapping strategies. The so-called equivalent capacity-based design invoking the 4-D signal space is used to determine the optimum coding rate of the STBC-SP-MLC scheme's component codes. Finally, we investigate a hybrid bit-to-SP symbol-mapping strategy for the STBC-SP-MLC scheme, which is capable of providing unequal error protection.
\end{abstract}

Index Terms-Equivalent capacity-based code design, multilevel coding, sphere-packing modulation, transmit diversity.

\section{INTRODUCTION}

$\mathbf{T}$ HE MULTILEVEL coding (MLC) concept in Fig. 1 was proposed by Imai and Hirawaki [1]. This scheme protects each bit of a nonbinary symbol with the aid of different-rate binary codes. Instead of using a high-complexity maximumlikelihood detector, the iterative multistage decoding (MSD) scheme (Fig. 1) was suggested in [1] to attain a high decoding performance at a low decoding complexity. The number of component codes in the MLC scheme (Fig. 1) depends on the number of coded bits, where the $i$ th bit constitutes the specific protection class of the constituent code $C^{i}$. This MSD process activates each different-rate component decoder one after the other, where each protection level exploits the a priori information obtained from the demodulator, before passing its extrinsic information to the $(i+1)$ st protection level associated with the constituent code $C^{i+1}$ in Fig. 1. This MLC scheme constitutes a flexible arrangement, where the grade of error protection can be adjusted for diverse applications.

To further improve the attainable performance of this MLC scheme, a substantial transmit diversity gain can be obtained

Manuscript received June 4, 2007; revised October 23, 2007 and November 26, 2007. This work was supported in part by the Engineering and Physical Sciences Research Council (EPSRC), U.K., by the Ministry of Higher Education of Saudi Arabia, and by the European Union under the auspices of the Phoenix and Newcom projects. The review of this paper was coordinated by Dr. C. Yuen.

The authors are with the School of Electronics and Computer Science, University of Southampton, SO17 1BJ Southampton, U.K. (e-mail: ryst03r@ ecs.soton.ac.uk; ora02r@ecs.soton.ac.uk; sxn@ecs.soton.ac.uk; 1h@ecs.soton. ac.uk).

Digital Object Identifier 10.1109/TVT.2008.915513 by introducing Alamouti's space-time block code (STBC) [2] when communicating over uncorrelated fading channels. Substantial research has been carried out in this area [3]-[5] by carefully designing both 2-D [3] and multidimensional (M-D) [5] bit-to-symbol partitioning schemes to improve the achievable bit error rate (BER) performance. Alamouti's concept of STBC was further generalized to an arbitrary number of transmitters by Tarokh et al. [6], but no attempt was made in [2] and [6] to jointly optimize the space-time signal design of the two consecutive time slots and two transmit antennas. In view of this, our main objective is to amalgamate the design of the spatial and time-domain signals with the aid of a novel spherepacking (SP) modulation scheme combined with orthogonal transmit diversity design [7].

Alamri et al. [8], [9] developed an SP soft demapper and introduced a turbo-detection-assisted SP-aided STBC design for the sake of achieving an iterative gain with the aid of an outer decoder. Motivated by these substantial performance improvements, in this treatise, we intrinsically amalgamate the SP and MLC concepts to create a novel orthogonal transmit diversity design. The minimum Euclidean distance (ED) of symbols defined in an M-D signaling constellation space may be maximized by finding the most meritorious mapping of the bits to the SP symbols. It is worth noting, however, that the choice of the best mapping typically depends on the channel conditions, as exemplified by Trellis-coded modulation arrangements designed for Gaussian channels [10], by bitinterleaved coded modulation schemes proposed for Rayleigh channels [10], or by the SP design of [8].

Due to the complex nature of bit-to-SP mapping in M-D SP modulation, we adopt a cost function (CF) based on maximizing the harmonic mean EDs between the different SP constellation points by employing the Binary Switching Algorithm (BSA) [11]. The SP constellation points associated with M-D SP schemes are usually categorized into different hierarchical layers according to their ED from the origin [12]. The multilayer SP constellation points of our scheme are constituted by a combination of Layer-1 and Layer-2 SP symbols to find the best cross-layer constellation scheme having the maximum minimum ED. This will be detailed in Section IV. Again, the bit-to-SP mapping can be optimized by applying the BSA to find the best $\mathrm{CF}$ value. The M-D SP symbols are then fed into the STBC encoder in Fig. 1. We refer to this scheme as an STBC-SP-MLC modulation arrangement.

A beneficial technique devised to determine each component code's rate in MLC was detailed by Wachsmann et al. [13], where the design concept exploited the so-called chain rule of mutual information introduced in [14]. Equivalent capacity 


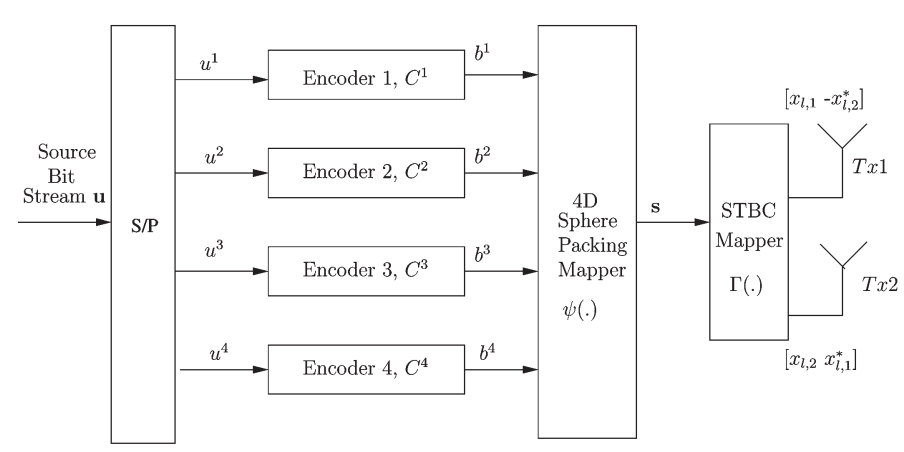

Transmitter

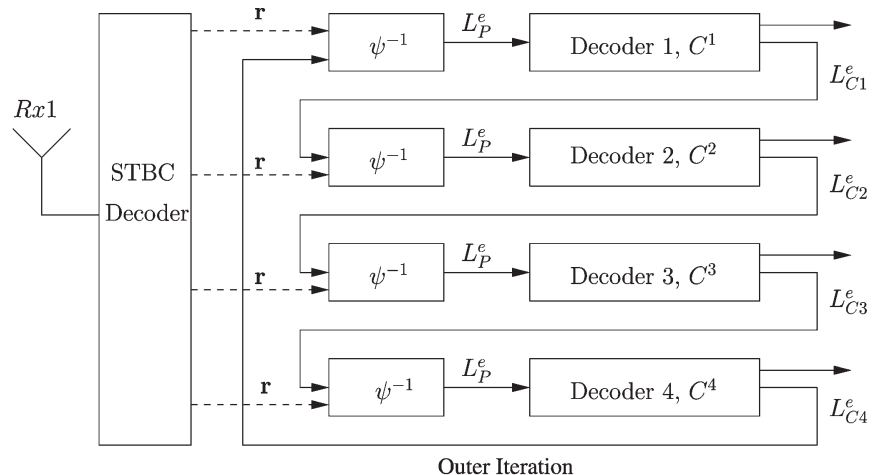

Receiver

Fig. 1. STBC-SP-MLC scheme.

rules were proposed for conventional 1-D and 2-D modulated signal constellations for transmission over additive white Gaussian noise, as well as Rayleigh fading channels. In this paper, we further extend the concepts proposed in [13] to improve the design of our STBC-SP-MLC scheme, invoking a 4-D SP constellation. More explicitly, the equivalent capacity design [13] will further be developed to determine the optimum low-density parity-check (LDPC) coding component code rates of the STBC-SP-MLC scheme, in conjunction with the various bit-to-SP symbol-mapping strategies in 4-D SP space.

To further deepen our discourse, we also design an unequal error protection (UEP) arrangement for our novel STBC-SPMLC scheme by proposing a novel hybrid partitioning by minimizing our $\mathrm{CF}$ with the aid of the BSA. The BER performance of the individual MLC protection classes of the amalgamated MLC scheme, as well as of the UEP hybrid scheme, will be evaluated by computer simulations when invoking the appropriate LDPC coding rates.

The novelty and rationale of the proposed system are as follows.

1) We create a novel turbo transceiver by amalgamating the constituent components of MLC with STBC in conjunction with SP modulation, which allows us to jointly capture time and space diversity with the aid of a new M-D SP modulation design, where the SP modulation maximizes the coding advantage of the transmission scheme by jointly designing and detecting the SP symbols hosting the two time slot's STBC symbols.

2) A novel equivalent capacity-based design is used to determine the coding rate of each MLC component code.

3) The BSA is used to create the optimum bit-to-SP mapping scheme by finding the best CF value. A UEP design is also proposed based on a hybrid mapping strategy.

This paper is organized as follows. In Section II, the outline of our STBC-SP-MLC turbo-transceiver is presented. The equivalent capacity-based design to determine the rate of the MLC component codes is detailed in Section III, whereas in Section IV, various mapping schemes are addressed. Our hybrid partitioning is introduced in Section $\mathrm{V}$ to create a UEP capability. This is followed by our simulation results in Section VI. Finally, we conclude in Section VII.

\section{SYSTEM OVERVIEW}

The schematic of the proposed STBC-SP-MLC turbotransceiver is shown in Fig. 1. The binary source bit stream $\mathbf{u}$ is serial-to-parallel converted at the transmitter. The four individual source bits, namely, $u^{1}, u^{2}, u^{3}$, and $u^{4}$, are protected by four different-rate MLC encoders, as shown in Fig. 1. The output bits of encoder $C^{i}$ are denoted as $b^{i}$, where $i=1,2,3$, and 4 .

We employ LDPC component codes owing to their powerful error-correcting capability, low complexity, and flexible coding rates. The random nature of the parity check matrix construction of LDPC codes allows us to dispense with the employment of additional channel interleavers. Each LDPC codeword is decoded using the belief propagation algorithm [15]. The MLCencoded bit substreams are then forwarded to the SP modulator $\psi$ in Fig. 1.

Here, we propose an M-D signal space modulation scheme, which is constructed using SP. The classic SP constellation points are packed as densely as possible, with a minimum amount of wasted space in between. Since a dense set of points is wanted, we use the SP $D_{4}$ [12], which is known to be the densest 4-D lattice having the highest ED in the realvalued 4-D Euclidean space $\mathbb{R}^{4}$ [12]. For a $D_{4}$ lattice, two adjacent centers must differ by at least one in two or more coordinate values or at least two in one coordinate value. This gives a minimum distance of $\sqrt{2}$ between the center of two spheres [12].

The 4-D SP phasor points are denoted as $S=\left(a_{l, 1}, a_{l, 2}\right.$, $\left.a_{l, 3}, a_{l, 4}\right)$, where we have $l=0,1,2, \ldots, M-1$, and $M$ is the number of SP constellation points. Here, we would like to represent the four individual coordinates of $S$ in 4-D SP space using real values while satisfying the $D_{4}$ constraint of $a_{l, 1}+$ $a_{l, 2}+a_{l, 3}+a_{l, 4}=k$, where $k$ is an even integer constant [12]. The total energy of the SP constellation points is represented by $E \triangleq \sum_{l=0}^{M-1}\left(a_{l, 1}^{2}+a_{l, 2}^{2}+a_{l, 3}^{2}+a_{l, 4}^{2}\right)[7]$.

Following SP modulation, the 4-D SP symbol is mapped to two complex-valued 2-bit symbols of a two-antenna STBC scheme. The bit-to-symbol mapping function of the system is denoted as [8] $\Gamma\left[\psi\left(b^{1}, b^{2}, b^{3}, b^{4}\right)\right]=\left\{a_{l, 1}+j a_{l, 2}, a_{l, 3}+\right.$ $\left.j a_{l, 4}\right\}=\left\{x_{l, 1}, x_{l, 2}\right\}$. Here, $\psi($.$) is the SP function used to map$ the original input bits to the SP symbols, and $\Gamma($.$) represents$ the mapping of the 4-D SP symbols to the complex-valued 2-bit symbols $x_{l, 1}$ and $x_{l, 2}$ after STBC encoding. 
Fig. 1 also shows the single-antenna-aided STBC receiver. The STBC decoder forwards its complex-valued symbols $\mathbf{r}$ to the SP demodulator $\psi^{-1}$ of Fig. 1, and the resultant bits are then decoded at different protection LDPC decoders in an iterative MSD manner. At the initial iteration, the SP demodulator $\psi^{-1}$ in Fig. 1 only receives the SP input symbol $S^{e}$ from the STBC decoder. The extrinsic logarithmic likelihood ratios (LLRs) $L_{P}^{e}$ produced by the SP demodulator are fed into the level-1 decoder of $C^{1}$, which then outputs a set of corresponding extrinsic LLRs $L_{C 1}^{e}$ to the demodulator. This LLR provides useful a priori information for the SP demodulator, where the LLRs gleaned from the previous MLC protection level are updated. As the decoding process continues, each MSD level receives useful a priori LLRs from the previous MSD level, which can be exploited in the LDPC decoder. The next outer iteration shown in Fig. 1 commences when the LLR information of the SP demodulator has been updated with the extrinsic information received from all MSD levels.

Alamri et al. [8] presented the concept of soft iterative SP demapping, in which the demapper receives LLRs $L_{C i}^{e}, i \in$ $\{1, \ldots, 4\}$ and provides the output LLR $L_{P}^{e}$ for the MLC outer codes. The details of the soft iterative demapper can be found in [8] and [16].

\section{EQUivalent CAPACITY DESIGN}

The technique of equivalent capacity-based code-rate design [13] relies on the maximization of the mutual information $I$ over all the relevant code parameters, exploiting the fact that the capacity of a particular channel can be formulated as $C=\max _{p\left(S_{i}\right)} I(R ; S)$, where $R$ denotes the legitimate received signal set, with $S$ representing the legitimate transmitted symbol set. We then apply the so-called chain rule of mutual information [13] as

$$
\begin{aligned}
I(R ; S)=I\left(R ; b^{1}\right)+I\left(R ; b^{2} \mid\right. & \left.b^{1}\right)+\cdots \\
& +I\left(R ; b^{q} \mid b^{1}, b^{2}, \ldots, b^{q-1}\right)
\end{aligned}
$$

where $b^{i}, i=1, \ldots, q$ denotes the individual binary bits of the different MLC protection levels. The so-called equivalent channel concept in [13] is based on the chain rule of (1), where each MLC protection level provides extrinsic information for the other bits and, hence, may be viewed as an independent "channel." This concept of having $q$ equivalent channels for bit $i$, where we have $q=\log _{2} M$, will be exploited in conjunction with the $a$ priori information gleaned from the previous $(i-1)$ protection levels, where the bits $b^{1}, \ldots, b^{i-1}$ have already been decoded and, hence, are assumed to be known. ${ }^{1}$

In the proposed STBC-SP-MLC scheme invoking 4-D SP modulation, each 4-D SP symbol is mapped to the complexvalued symbols $x_{l, 1}$ and $x_{l, 2}$ before being mapped to the two consecutive timeslots associated with the two STBC transmit antennas, as shown in Fig. 1. The resultant signal is then transmitted over a correlated Rayleigh fading channel. These

\footnotetext{
${ }^{1}$ The information provided by the bits of a nonbinary symbol for each other may be interpreted as additional auxiliary information provided by a fictitious channel, which is also termed the equivalent channel in [13].
}

STBC symbols $x_{l, 1}$ and $x_{l, 2}$ have an unequal probability of occurrence within the resultant 4-bit SP signal constellation points, as will be shown in the context of Table II. The partitioning of the SP signal set $S$ can be further divided into two parts, resulting in the SP subsets labeled $S\left(b^{1}=0\right)$ and $S\left(b^{1}=1\right)$, each containing a total of eight out of the $M=16$ $\mathrm{SP}$ symbols. In each SP subset, for example, at the $i$ th level of the SP subset $S\left(b^{1}=0\right)$, the eight-symbol SP constellation segment can further be subdivided into two four-symbol SP subsets labeled $S\left(b^{1}=0, b^{2}=0\right)$ and $S\left(b^{1}=0, b^{2}=1\right)$ at MLC level $(i+1)$, etc. The partitioning tree of the SP signal set is completed when the partitioned SP constellation subset contains only a single SP symbol at level $q$.

For a 2-D STBC scheme having $N_{t}=2$ transmitter and $N_{r}=1$ receiver antennas, the signal $R$ received at the single antenna can be represented as [17], [18]

$$
R=\sum_{j=1}^{N_{t}}\left|h_{j}\right|^{2} X+\Omega=\chi_{2 N t}^{2} X+\Omega
$$

where $X$ is the 2-D complex-valued received signal, $h_{j}$ represents the complex-valued Rayleigh fading coefficient, and $\chi_{2 N t}^{2}$ represents a chi-square-distributed random variable having $2 N_{t}$ degrees of freedom. Furthermore, $\Omega$ denotes the resultant equivalent noise at the STBC receiver having zero mean and a variance of $\chi_{2 N_{t}}^{2} N_{0} / 2$ per dimension, where $N_{0} / 2$ is the original noise variance per dimension.

The STBC-SP-MLC system (Fig. 1) characterized in (2) receives two complex-valued STBC symbols of the signal $R$ and transmits the $M$-ary 2-D STBC signals $X_{m}, m \in$ $\{1,2, \ldots, M\}$, over the two STBC antennas in two consecutive timeslots. Given a general $J$-dimensional received signal $R$, provided that the $J$-dimensional $M$-ary signal $X$ was transmitted, (2) can be generalized as [17]

$$
R[d]=\chi_{2 N_{t}}^{2}[d] X[d]+\Omega[d]
$$

where $d$ refers to the dimension index of the signal, whereas $R=(R[1], \ldots, R[J]), X=(X[1], \ldots, X[J])$, and $\Omega=(\Omega[1], \ldots, \Omega[J])$. When $J \geq 2$ and even, we have $J / 2$ $\chi_{2 N_{t}}^{2}$ components because $\chi_{2 N_{t}}^{2}[k]=\chi_{2 N_{t}}^{2}[k+1]$ for $k \in$ $\{1,3,5, \ldots\}$ represents the complex-valued channel having two dimensions.

The conditional probability density function (pdf) of receiving a $J$-dimensional signal $R$, given the $J$-dimensional $M$-ary transmitted signal $X$, is expressed as [17]

$$
\begin{aligned}
p & \left(R \mid X, \chi_{2 N_{t}}^{2}\right) \\
& =\prod_{d=1}^{J} \frac{1}{\sqrt{\pi N_{0 \chi_{2 N_{t}}^{2}[d]}}} \exp \left(\frac{-\left|R[d]-\chi_{2 N_{t}}^{2}[d] X[d]\right|^{2}}{\chi_{2 N_{t}}^{2}[d] N_{0}}\right) \\
= & \frac{1}{\prod_{d=1}^{J} \sqrt{\pi N_{0 \chi_{2 N_{t}}^{2}[d]}}} \exp \left(\sum_{d=1}^{J} \frac{-\left|R[d]-\chi_{2 N_{t}}^{2}[d] X[d]\right|^{2}}{\chi_{2 N_{t}}^{2}[d] N_{0}}\right) .
\end{aligned}
$$

The corresponding conditional pdf of one of the 2-D $M$-ary complex-valued received components at each of the 
STBC antennas is given by

$$
p\left(R \mid X, \chi_{2 N_{t}}^{2}\right)=\frac{1}{\pi N_{0} \chi_{2 N_{t}}^{2}} \exp \left(\frac{-\left|R-\chi_{N_{t}}^{2} X\right|^{2}}{\chi_{2 N_{t}}^{2} N_{0}}\right) .
$$

In contrast to conventional modulation, where the maximum achievable capacity is attained when each constellation point has an equal probability of occurrence $1 / M$, the probability of occurrence of all legitimate transmitted $M$-ary signals $X_{m}$, where $m$ is the symbol index of $m \in$ $\{1, \ldots, M\}$, is, in this case, different. This means that at MLC partitioning level $i$, each legitimate 2-D STBC constellation point will be transmitted with a different relative frequency at each transmit antenna. Each of them has a probability of $p\left(X_{m}\right)$ for $m \in\{1,2, \ldots, M\}$, and the mutual information between $R$ and $X$ [14] can be expressed as

$$
\begin{aligned}
I(X ; R)= & \sum_{m=1}^{M} \int_{R} p\left(X_{m}, R\right) \log _{2}\left(\frac{p\left(X_{m}, R\right)}{p\left(X_{m}\right) p(R)}\right) d R \\
= & \sum_{m=1}^{M} \int_{R} p\left(R \mid X_{m}\right) p\left(X_{m}\right) \\
& \cdot \log _{2}\left(\frac{p\left(R \mid X_{m}\right)}{\sum_{n=1}^{M} p\left(R \mid X_{n}\right) p\left(X_{n}\right)}\right) d R .
\end{aligned}
$$

Expressing the mutual information with the aid of the entropy as $I(X ; R)=H(X)-H(X \mid R)$, we arrive at [17]

$$
\begin{aligned}
& I(X ; R)=-\sum_{m=1}^{M} p\left(X_{m}\right) \log _{2}\left(p\left(X_{m}\right)\right) \\
& \quad-\sum_{m=1}^{M} p\left(X_{m}\right) E\left[\log _{2}\left(\frac{p\left(X_{n}\right)}{p\left(X_{m}\right)} \sum_{n=1}^{M} \exp \left(\Psi_{m, n}\right)\right) \mid X_{m}\right]
\end{aligned}
$$

where we have $\Psi_{m, n}=-\left|\chi_{2 N_{t}}^{2}\left(X_{m}-X_{n}\right)+\Omega\right|^{2}+|\Omega|^{2} /$ $\chi_{2 N_{t}}^{2} N_{0}$, and $E\left[A \mid X_{m}\right]$ is the expectation of $A$ conditioned on $X_{m}$.

The STBC-SP-MLC scheme uses a single receive and two transmit antennas, and there are two modulated STBC symbols, each gleaning the amount of mutual information quantified by (7). At the first partitioning level, where there are two partitioning branches labeled $S\left(b^{1}=0\right)$ and $S\left(b^{1}=1\right)$, the total mutual information between a transmitted 4-D SP symbol and the received 4-D SP symbol is the average of that of the two partitioning branches of the 2-D STBC symbols.

To obtain the mutual information for a 2-D STBC symbol at partitioning level $i$, given the perfect knowledge of all the previous $1, \ldots, i-1$ partitioning levels and a total of $q$ partitioning levels, we express (7) as

$$
I\left(\mathbf{R}^{\mathbf{i}} ; \mathbf{X}^{\mathbf{i}}\right)=I\left(\mathbf{R}^{\mathbf{i}} ; \mathbf{b}^{\mathbf{i}}, \ldots, \mathbf{b}^{\mathbf{q}} \mid \mathbf{b}^{\mathbf{1}}, \ldots, \mathbf{b}^{\mathbf{i}-\mathbf{1}}\right)
$$

where $\mathbf{R}^{\mathbf{i}}$ and $\mathbf{X}^{\mathbf{i}}$ denote the received and transmitted STBC symbols at partitioning level $i$, respectively. The total number of constellation points $M$ in (7) will be reduced according to the SP constellation points at the particular partitioning level $i$.
TABLE I

FIRST TWO LAYERS OF $D_{4}$

\begin{tabular}{|c|c|c|c|c|c|c|}
\hline Layer & \multicolumn{4}{|c|}{ Constellation Points } & Norm & Number of Combinations \\
\hline \hline 0 & 0 & 0 & 0 & 0 & 0 & 1 \\
\hline \hline 1 & $+/-1$ & $+/-1$ & 0 & 0 & 2 & 24 \\
\hline \hline 2 & $+/-2$ & 0 & 0 & 0 & 4 & 8 \\
\cline { 2 - 7 } & $+/-1$ & $+/-1$ & $+/-1$ & $+/-1$ & 4 & 16 \\
\hline
\end{tabular}

TABLE II

Bit MAPPINGS FOR DiFFERENT SCHEMES DESIGNED FOR STBC-SP WITH $M=16$

\begin{tabular}{|c|c|c|c|c||c|c|c|c|}
\hline Index & \multicolumn{2}{|c|}{ L-1 } & \multicolumn{1}{l||}{ and $\mathrm{L}-2$ from $D_{4}$} & \multicolumn{5}{|c|}{ Sym Index, $S_{i}=j, i \in\{0 \ldots 15\}$} \\
\hline$j$ & $a_{1}$ & $a_{2}$ & $a_{3}$ & $a_{4}$ & SET & L1-BSA & L2-BSA & UEP1 \\
\hline 0 & -1 & -1 & 0 & 0 & 1 & 0 & 3 & 6 \\
\hline 1 & 0 & -1 & -1 & 0 & 0 & 5 & 12 & 4 \\
\hline 2 & 0 & -1 & +1 & 0 & 8 & 2 & 5 & 5 \\
\hline 3 & +1 & -1 & 0 & 0 & 9 & 15 & 0 & 7 \\
\hline 4 & -1 & 0 & 0 & +1 & 2 & 13 & 15 & 13 \\
\hline 5 & 0 & 0 & -1 & +1 & 12 & 6 & 9 & 15 \\
\hline 6 & 0 & 0 & +1 & +1 & 4 & 4 & 10 & 2 \\
\hline 7 & +1 & 0 & 0 & +1 & 5 & 10 & 6 & 10 \\
\hline 8 & -1 & 0 & 0 & -1 & 14 & 12 & 16 & 8 \\
\hline 9 & 0 & 0 & -1 & -1 & 15 & 11 & 17 & 0 \\
\hline 10 & 0 & 0 & +1 & -1 & 7 & 14 & 18 & 11 \\
\hline 11 & +1 & 0 & 0 & -1 & 6 & 1 & 19 & 9 \\
\hline 12 & -1 & +1 & 0 & 0 & 13 & 3 & 20 & 14 \\
\hline 13 & 0 & +1 & -1 & 0 & 3 & 8 & 21 & 12 \\
\hline 14 & 0 & +1 & +1 & 0 & 11 & 9 & 22 & 1 \\
\hline 15 & +1 & +1 & 0 & 0 & 10 & 7 & 23 & 3 \\
\hline 16 & -2 & 0 & 0 & 0 & - & - & - & - \\
\hline 17 & 0 & 0 & -2 & 0 & - & - & - & - \\
\hline 18 & 2 & 0 & 0 & 0 & - & - & - & - \\
\hline 19 & 0 & 2 & 0 & 0 & - & - & - & - \\
\hline 20 & 0 & -2 & 0 & 0 & - & - & - & - \\
\hline 21 & 0 & 0 & 2 & 0 & - & - & - & - \\
\hline 22 & 0 & 0 & 0 & 2 & - & - & - & - \\
\hline 23 & 0 & 0 & 0 & -2 & - & - & - & - \\
\hline & & & & & & & & \\
\hline
\end{tabular}

Finally, the information gleaned at MLC protection level $i$ can be calculated from the chain rule of (1) according to [13]

$$
\begin{gathered}
I\left(\mathbf{R} ; \mathbf{b}^{\mathbf{i}} \mid \mathbf{b}^{\mathbf{1}}, \ldots, \mathbf{b}^{\mathbf{i}-\mathbf{1}}\right)=I\left(\mathbf{R} ; \mathbf{b}^{\mathbf{i}}, \ldots, \mathbf{b}^{\mathbf{q}} \mid \mathbf{b}^{\mathbf{1}}, \ldots, \mathbf{b}^{\mathbf{i}-\mathbf{1}}\right) \\
-I\left(\mathbf{R} ; \mathbf{b}^{\mathbf{i}+\mathbf{1}}, \ldots, \mathbf{b}^{\mathbf{q}} \mid \mathbf{b}^{\mathbf{1}}, \ldots, \mathbf{b}^{\mathbf{i}}\right) .
\end{gathered}
$$

\section{MAPPING Design}

The legitimate constellation points hosted by $D_{4}{ }^{2}$ of the SP scheme are categorized into layers based on their norms or energy, i.e., the distance from the origin. The objective of mapping design is to assign each signal label to different SP points of $D_{4}$. We investigate the first two layers of the $D_{4} \mathrm{SP}$ constellation, which are characterized in Table I. At Layer-1, the SP symbol centered at $(0,0,0,0)$ has a total of 24 closestneighbor SP symbols around it, which are constituted by the various legitimate combinations of $( \pm 1, \pm 1,0,0)$, as shown in Table II.

For a 4-D SP symbol belonging to a constellation size of $M=16$, the effective throughput $\tau$ of the STBC-SP arrangement using no outer MLC encoder is expressed as

$$
\begin{aligned}
\tau & =1 \text { symbol } /(2 \text { channel use }) \times 4 \text { bits } / \text { symbol } \\
& =2 \text { bits } /(\text { channel use }) .
\end{aligned}
$$

\footnotetext{
${ }^{2} D_{4}$ is a 4-D lattice having the best Euclidean distance in the 4-D real Euclidean space $\mathbb{R}^{4}[12]$.
} 
For the sake of fair comparison, the uncoded 2-bit/symbol QPSK benchmarker is used because it has the same throughput of $\tau=2 \mathrm{bits}$ (channel use) as that in (10). Since there are 24 immediately adjacent SP neighbors at Layer-1, which have different EDs in the 4-D SP constellation [12], we select the specific $M=16$ points that exhibit maximum EDs. These $M=16$ points that we selected using exhaustive computer search from the $24 \mathrm{SP}$ points of $D_{4}$ are shown in Table II, which have the maximum ED between the adjacent neighboring points at a given energy.

The first set of bit-to-SP mapping or labeling based on this constellation space was obtained by an exhaustive computer search by maximizing the summed ED between the pair of SP symbols belonging to a specific partitioning labeled by the MLC bits, which we term as SET (as in set partitioning). The resultant SP assignment is shown in Table II. Then, the BSA [11] is utilized to design different bit-to-SP mapping schemes. Our bit-to-SP mapping CF is the reciprocal of the squared ED of each SP symbol with respect to other SP symbols within a specific partitioned subset, as labeled by a particular MLC bit. The CF can be described as

$$
C F=\sum_{i=1}^{q} \sum_{b=0}^{1} \sum_{S_{k} \in \Gamma_{b}^{i}} \frac{1}{\left|S_{k}-\hat{S}_{k}\right|^{2}} .
$$

We use the simplifying assumption here that the SP demapper has perfect a priori information, which constitutes a good approximation at high signal-to-noise ratios, and that the SP signal space has $M=2^{q}$ constellation points. The notation $\Gamma$ represents the SP-to-STBC symbol-mapping function, which maps the bits $b \in\{0,1\}$ to the SP symbols $S_{k}$. The symbol $\hat{S}_{k}$ has the same binary bit label as $S_{k}$, except for the $i$ th bit.

By minimizing the CF of (11) using the BSA, the specific partitioning corresponding to the maximum harmonic mean of the ED $[11]^{3}$ for the SP constellation points belonging to the opposite bit values at the same SP partitioning level in the entire set of mapping schemes is chosen. We term this a Layer-1 BSA (L1-BSA) mapping, and the corresponding symbol indices are given in Table II.

As another design alternative, we further extend the constellation by additionally incorporating SP symbols from the Layer-2 constellation space. Observe from Table I that similar to Layer-1, there are also a total of 24 points on Layer-2 [12]. In our second candidate scheme, we select the eight highest-distance SP symbols from Layer-1 as well as from Layer-2 to form a new SP constellation. By using the CF of (11), we obtain a different mapping for this constellation. We call this the BSA Layer-2-aided (L2-BSA) scheme, which is also characterized in Table II.

The index $j$ shown in the first column of Table II identifies the constellation points selected from both Layer-1 and Layer-2 after finding the specific points having the maximum ED in the search space $D_{4}$. Note that the Layer-1 points span from the index $i=0$ to 15 , indicating the 16 points of Layer-1, whereas the Layer- 2 points span from the index $i=16$ to 23 . The

\footnotetext{
${ }^{3}$ Harmonic mean distance is the reciprocal of the arithmetic average of the inverse distance in the set.
}

notation $S_{i}=j$ indicates that the SP symbol $S_{i}$ is assigned to the constellation point of index $j$ in Table II. The index $i$ associated with symbol $S_{i}$ is assigned in increasing order from " 0 " to " 15 ." For example, referring to the L1-BSA mapping scheme of Table II, the first three rows are 0, 5, and 2, which indicate the SP symbols of $S_{0}, S_{1}$, and $S_{2}$. These symbols are associated with the indices of 0,5 , and 2 , which correspond to the constellation points of $(-1,-1,0,0),(0,0,-1,+1)$, and $(0,-1+1,0)$, respectively.

\section{UEP}

The STBC-SP-MLC system proposed here may also be studied in comparison with the STBC-SP scheme concatenated with a single-class outer encoder. The ability to provide UEP is important for various speech and video applications, where each bit may have a different error sensitivity. The MLC scheme is capable of providing UEP for high-quality, error-resilient speech and video transmissions.

To equip our STBC-SP-MLC scheme with an UEP capability [19], we invoke a hybrid SP constellation-partitioning approach. Instead of maximizing the ED of SP symbols corresponding to all bit-level symbols, this hybrid partitioning will tolerate lower distance SP symbols labeled by vulnerable bits while enhancing the protection of other selected bits by more widely spacing their SP symbols.

This is achieved by creating an unequal distance-based mapping strategy. The first partitioning level of $i=1$ now minimizes rather than maximizes the ED between the signal points, which creates one of the more vulnerable MLC protection classes. By minimizing the power assigned to $i=1$, we are able to expand the ED of other UEP classes by assigning a higher power to them. For the rest of the STBC-SP-MLC UEP classes at $i=2,3,4$, the classic Ungerböck partitioning (UP) concept is applied [20], which minimizes the ED between the SP signal points.

The optimization problem to be solved is to minimize the ED at the MLC level $i=1$ and to maximize it at levels $i=2,3$, and 4 by formulating the following $\mathrm{CF}$ :

$$
\begin{aligned}
\mathrm{CF}=\sum_{i=1}^{q} \sum_{b=0}^{1}\left[\sum_{S_{k} \in \Gamma_{b}^{i}, i=1}\left\|S_{k}-\hat{S_{k}}\right\|^{2}\right. & \\
& \left.+\sum_{S_{k} \in \Gamma_{b}^{i}, i \neq 1}\left\|S_{k}-\hat{S}_{k}\right\|^{-2}\right]
\end{aligned}
$$

which is minimized again using the BSA [11]. As before, we assume here that the SP demapper has perfect a priori information, i.e., the decoder in Fig. 1 used a sufficiently high number of iterations to have an infinitesimally low BER, and that the SP signal space has $M=2^{q}$ constellation points. Again, the notation $\Gamma$ represents the SP-to-STBC symbolmapping function, which maps the bits $b \in\{0,1\}$ to the SP symbols $S_{k}$. As before, the symbol $\hat{S}_{k}$ is the symbol in 4-D SP space, which is mapped according to $\Gamma_{\bar{b}}^{i}$, where $\bar{b}$ denotes the complement of bit $b$ at bit index $i$. Having designed the bit-to-SP symbol mapping, the aforementioned equivalent 
TABLE III

SYSTEM PARAMETERS

\begin{tabular}{|l|r|}
\hline Sphere packing modulation & $M=16$ \\
\hline Conventional modulation & QPSK \\
\hline MLC component output block & 640 bits \\
\hline STBC-SP-LDPC output block & 2560 bits \\
\hline No. of LDPC iterations & 5 \\
\hline LDPC column weight & 3 \\
\hline Channel & Correlated Rayleigh Fading \\
\hline Normalised Doppler frequency & 0.1 \\
\hline Throughput & \\
STBC-SP $M=16$ & (without outer code) \\
STBC-QPSK & 2 bits/channel use \\
\hline System-1 & STBC-SP-MLC, L1-BSA \\
System-2 & STBC-SP-MLC, L2-BSA \\
System-3 & STBC-QPSK-MLC, UP \\
System-4 & STBC-SP-MLC, UEP \\
System-5 & STBC-SP-LDPC(2560, 1280), SET \\
\hline
\end{tabular}

capacity-based code-rate calculation for L1-BSA mapping is used to maintain the specific coding rate at each UEP MLC level. The corresponding mapping scheme is shown in Table II. We term this new mapping strategy UEP1. We summarize all our proposed systems in Table III for convenient comparison.

\section{RESUlTs AND Discussion}

In this section, we employ LDPC codes as MLC component codes, and the rest of the parameters are summarized in Table III. The complexity of the STBC-SP-MLC encoder depends on the LDPC component codes, where the encoder's complexity increases as a quadratic function of the block length noted as $O\left(N^{2}\right)$, where $N$ is the block length in bits. At the receiver, the complexity is also dominated by that of the LDPC decoders, since both the STBC decoder and the SP demapper impose a negligible complexity. The overall decoding complexity associated with the detection of each LDPC-coded bit in a single iteration is $4 w_{c}$ additions and $14 w_{c}$ multiplications, where $w_{c}$ is the column weight [21] of the LDPC parity check matrix. Hence, in our STBC-SP-MLC scheme, which has a three-column weight, uses five inner LDPC iterations, and generates 640 LDPC-encoded bits, the decoder's complexity per MLC decoder having four LDPC-encoded blocks becomes $4 \times 3 \times 640 \times 4 \times 5 \times(I+1)=153600(I+1)$ additions and $14 \times 3 \times 640 \times 4 \times 5 \times(I+1)=537600(I+1)$ multiplications, where $I$ denotes the number of outer iterations.

Taking an example of the SET mapping scheme of STBCSP-MLC, we use the equivalent capacity-based rate calculation of [13] and note that the STBC symbol $\left(a_{1}+j a_{2}\right)$ is mapped to the first STBC transmitter of Fig. 1, whereas $\left(a_{3}+j a_{4}\right)$ is mapped to the second transmitter. All the legitimate combinations of the $\left(a_{1}, a_{2}\right)$ and $\left(a_{3}, a_{4}\right)$ values are plotted in Fig. 2. We have a total of nine visibly different legitimate constellation points in Fig. 2, because some of the points are identical, as suggested by the associated doubled or quadrupled probability of occurrence. For example, observe in Table II for the mapping scheme SET that the probability of the constellation point $(-1,0)$, which corresponds to $S_{2}$ and $S_{6}$ for the first transmitter $T \times 1$, is calculated as $2 / 16=0.125$. Similarly, the

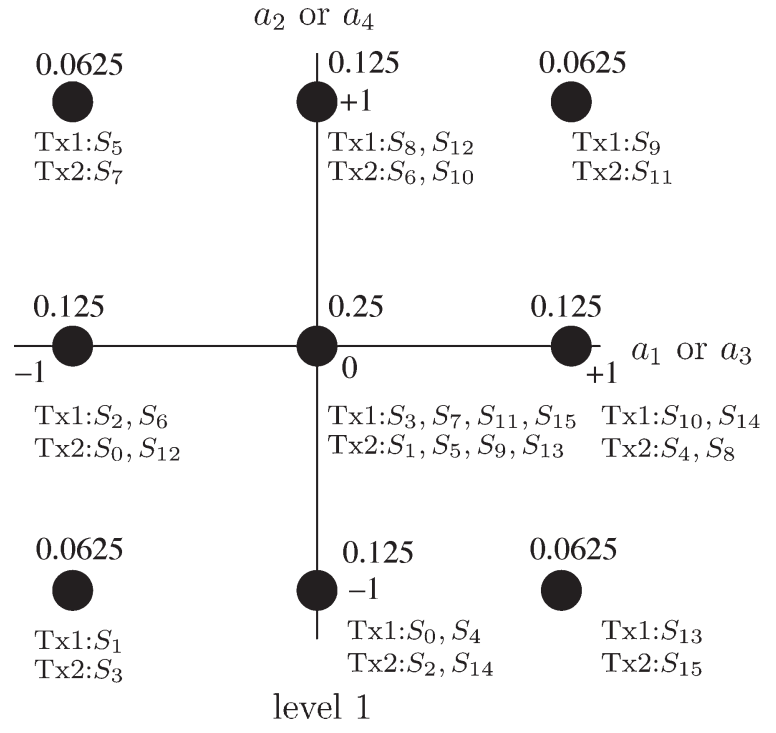

Fig. 2. STBC constellation points of SET mapping scheme (System-1) outlined in Table II at level 1 . The number above the dots indicates the probability of occurrence for the symbols, whereas the symbol indices at the bottom indicate the specific symbol. The first and second transmitters are represented by $T \times 1$ and $T \times 2$, respectively.

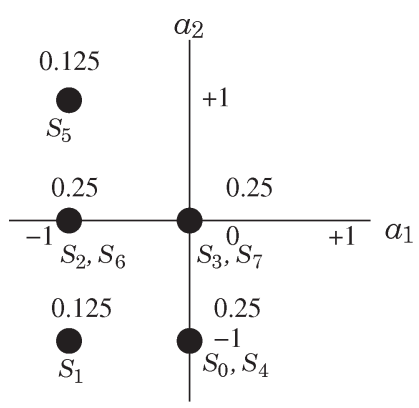

(a)

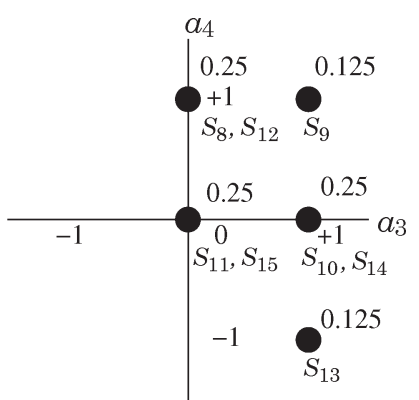

(b)
Fig. 3. Constellation points of the first STBC transmitter $T \times 1$ at MLC level 2 based on the SET mapping scheme (System-1) outlined in Table II. The number above the dots indicates the probability of occurrence for the symbols, whereas the symbol indices at the bottom indicate the specific symbol. (a) Level $2, b^{1}=0$. (b) Level $2, b^{1}=1$.

probability of occurrence for all the specific constellation points is indicated by the number written above each point in Fig. 2.

At the next Level-2 MLC protection class, the signal representing the first STBC symbol of transmitter 1 is shown in Fig. 3(a) and (b). Again, here, we denote each 2-D STBC symbol as $X$. The resultant five-point subsets $X\left(b^{1}=0\right)$ and $X\left(b^{1}=1\right)$ provide us with a partition tree of $X\left(0 b^{2}, \ldots, b^{q}\right)$ and $X\left(1 b^{2}, \ldots, b^{q}\right)$. Given the knowledge of bit $b^{1}$ at level 2, which identifies one of the two partitions shown in Fig. 3, we obtain the partitioning of $X\left(b^{2}, \ldots, b^{q} \mid b^{1}\right)$ at level 2 of the first transmitter. The two branches resulting from this partitioning yield the five unequal-probability constellation points shown in Fig. 3. The partitioning process continues from level 1 to level $q$. Since, in the context of MSD, we assume having virtually independent channels for each protection level, the mutual information inferred at each protection level $i$ can be calculated from (7)-(9).

Fig. 4 shows the equivalent capacity curves detailed in Section III for the L1-BSA mapping scheme. The vertical 


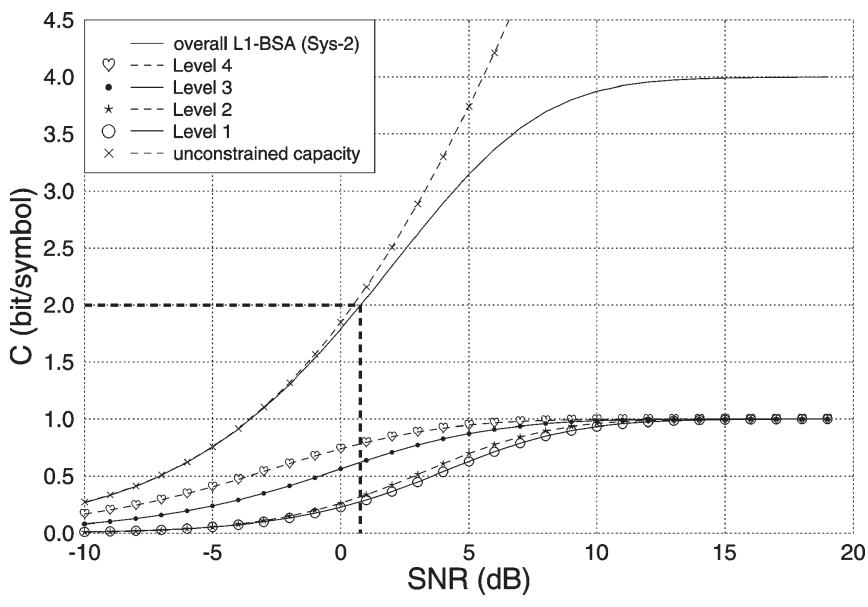

Fig. 4. Equivalent capacity curves of the proposed SP-STBC with L1-BSA mapping scheme (System-1) communicating over Rayleigh fading channel, where an STBC scheme having $N_{t}=2$ and $N_{r}=1$ antennas was used.

TABLE IV

CODING RATES AND SP GAINS OF STBC-SP-MLC AND STBC-QPSK-MLC Schemes. The SP Gains ARe the Modulation Gains for Both SYSTEM-1 AND SYSTEM-2, AS COMPARED WiTH THAT OF THE BENCHMARKER STBC-QPSK-MLC (SYSTEM-3) AT BER $10^{-5}$, INVOKING ITERATION $I=0$ AND $I=4$, RESPECTIVELY

\begin{tabular}{|c||c|c|c|c|}
\hline Coding rate & $R_{1}$ & $R_{2}$ & $R_{3}$ & $R_{4}$ \\
\hline \hline $\begin{array}{c}\text { STBC-SP-MLC } \\
\text { L1-BSA } \\
\text { (System-1) }\end{array}$ & $\begin{array}{c}0.2717 \\
(176 / 640)\end{array}$ & $\begin{array}{c}0.3152 \\
(204 / 640)\end{array}$ & $\begin{array}{c}0.6196 \\
(398 / 640)\end{array}$ & $\begin{array}{c}0.7826 \\
(502 / 640)\end{array}$ \\
\hline $\begin{array}{c}\text { STBC-SP-MLC } \\
\text { L2-BSA } \\
\text { (System-2) }\end{array}$ & $\begin{array}{c}0.3043 \\
(195 / 640)\end{array}$ & $\begin{array}{c}0.3913 \\
(250 / 640)\end{array}$ & $\begin{array}{c}0.5109 \\
(327 / 640)\end{array}$ & $\begin{array}{c}0.7935 \\
(508 / 640)\end{array}$ \\
\hline $\begin{array}{c}\text { STBC-QPSK-MLC } \\
\text { Ungerböck part. } \\
\text { (System-3) }\end{array}$ & $\begin{array}{c}0.6641 \\
(425 / 640)\end{array}$ & $\begin{array}{c}0.3359 \\
(215 / 640)\end{array}$ & - & - \\
\hline \hline SP gain & \multicolumn{3}{|c|}{ at BER=10-5 in $E_{b} / N_{0}[\mathrm{~dB}]$} \\
\cline { 2 - 5 } & \multicolumn{2}{|c|}{$I=0$} & \multicolumn{2}{c|}{$I=4$} \\
\hline System-1 & \multicolumn{2}{|c|}{4.08} & 3.16 \\
\hline System-2 & \multicolumn{2}{|c|}{3.75} & \multicolumn{2}{c|}{} \\
\hline
\end{tabular}

dashed line at the throughput of $2 \mathrm{bit} / \mathrm{symbol}$ is plotted to determine the equivalent capacity for each protection level of the MLC scheme. Since the total throughput of the SP-STBCMLC arrangement for $M=16$ is $2 \mathrm{bit} / \mathrm{symbol}$ or $1 \mathrm{bit} / \mathrm{channel}$ use, the throughput of the individual different protection subchannels will sum up to be the same as the overall SP-STBCMLC scheme's throughput. The coding rates determined from the equivalent capacity rules outlined at the end of Section III are $(0.2717,0.3152,0.6196,0.7826)$. The actual LDPC code rates for the rest of the mapping schemes using the same calculation method are shown in Table IV.

A total of 5000 frames containing 2560 MLC-encoded bits were transmitted for the sake of our BER evaluation. We construct the STBC-SP-MLC scheme with a total MLC coding rate of $1 / 2$, giving the overall system throughput of $(2$ bits/channel use $\times 1 / 2)=1$ bit/channel use. Our benchmarker is based on an STBC-QPSK-MLC (System-3) structure, which is constituted by the direct serial concatenation of STBC and MLC with the identical QPSK scheme. The STBC employs two transmit antennas and a single receive antenna, and the MLC maps its encoded symbols to a 2-D UP-based QPSK arrangement. The overall MLC coding rate for this

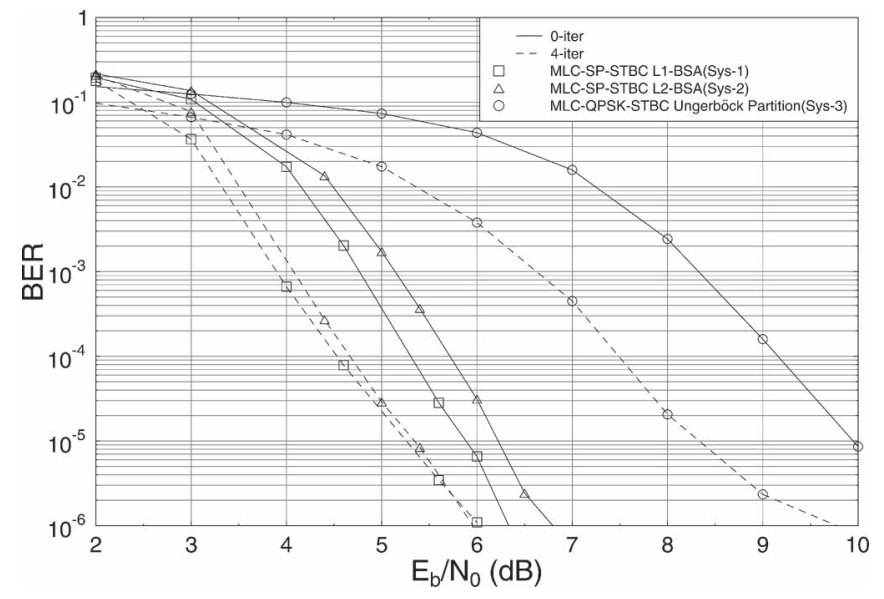

Fig. 5. BER versus $E_{b} / N_{0}$ performance of the STBC-QPSK-MLC scheme (System-3) at an effective throughput of 1 bit/symbol using UP-based bit-tosymbol mapping and our proposed STBC-SP-MLC scheme using L1-BSA (System-1) and L2-BSA mapping (System-2) strategies, when communicating over a correlated Rayleigh channel having a Doppler frequency of 0.1. All other parameters are summarized in Tables III and IV.

STBC-QPSK-MLC benchmarker is also $1 / 2$, giving an overall system throughput of $(2$ bits/channel use $\times 1 / 2)=$ $1 \mathrm{bit} / \mathrm{channel}$ use. The L1-BSA (System-1) and L2-BSA (System-2) mapping schemes are characterized in Fig. 5.

Conventional MSD-aided MLC does not perform well in a Rayleigh fading channel, owing to the potential interlayer error propagation [4], although the spatial diversity gain provided by a serially concatenated STBC scheme usefully improves its BER performance. The BER curve reaches $10^{-5}$ using four iterations at $E_{b} / N_{0}=8.3 \mathrm{~dB}$. However, by invoking the proposed SP demapper, the BER dips below $10^{-5}$ at $E_{b} / N_{0}=$ $5.15 \mathrm{~dB}$ upon using four iterations. By further expanding the SP constellation to the Layer-2 $D_{4}$ space, the BER performance reaches $10^{-5}$ at around $E_{b} / N_{0}=5.35 \mathrm{~dB}$. However, both schemes exhibit a similar BER of about $10^{-6}$ at $E_{b} / N_{0}=6 \mathrm{~dB}$.

Finally, we also constructed a single-class $1 / 2$-rate STBCSP-LDPC benchmark scheme having an effective throughput of $1 \mathrm{bit} / \mathrm{channel}$ use for comparison with our MLC scheme, where the MLC code is replaced by a single-class $\operatorname{LDPC}(2560,1280)$ scheme having a coding rate of $1 / 2$. All the LDPC component codes employed in our simulations used a total of five iterations to generate sufficiently reliable extrinsic LLRs. The complexities of a single 2560-coded-bit LDPC code and that of the four 640-coded-bit MLC-LDPC component codes of Table IV were deemed similar in these systems. Our proposed STBCSP-MLC (System-1) exhibits a similar BER performance to that of the single-class STBC-SP-LDPC (System-4) structure characterized in Fig. 6, although the proposed scheme has a slightly better performance at a low number of iterations. By contrast, the single-class scheme of Fig. 6 performs approximately $0.3 \mathrm{~dB}$ better when using a higher number of iterations at $\mathrm{BER}=10^{-5}$.

This is a consequence of the fact that each MLC component code has a four-times lower codeword length compared with the single-class $\operatorname{LDPC}(2560,1280)$ code. Therefore, the BER performance of the STBC-SP-MLC (System-1) scheme can be improved by increasing the block length of each LDPC 


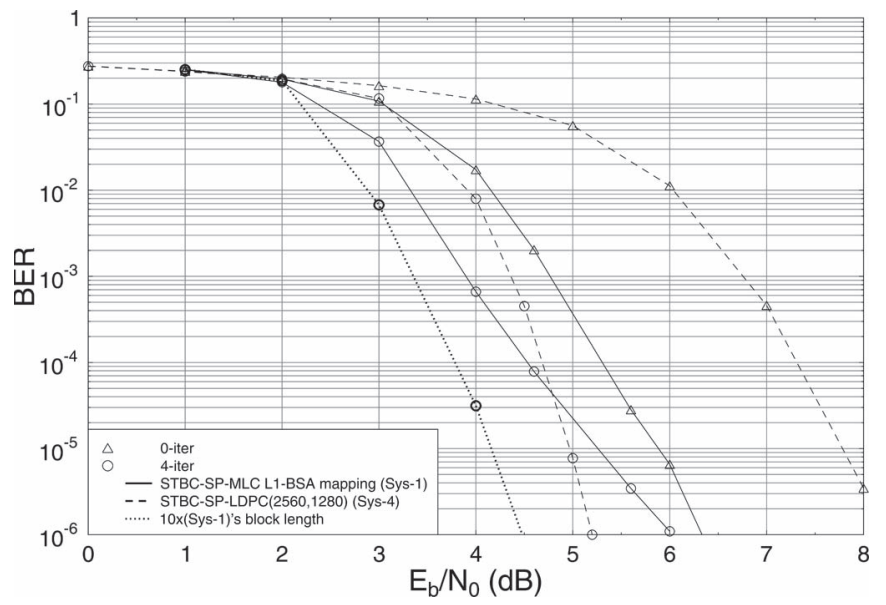

Fig. 6. BER versus $E_{b} / N_{o}$ performance of the proposed STBC-SP-LDPC scheme (System-4) using a single 1/2-rate component code LDPC $(2560,1280)$ and having an effective throughput of $1 \mathrm{bit} / \mathrm{channel}$ use, in comparison with the proposed STBC-SP-MLC scheme (System-1), when communicating over a correlated Rayleigh fading channel. All other parameters are summarized in Tables III and IV.

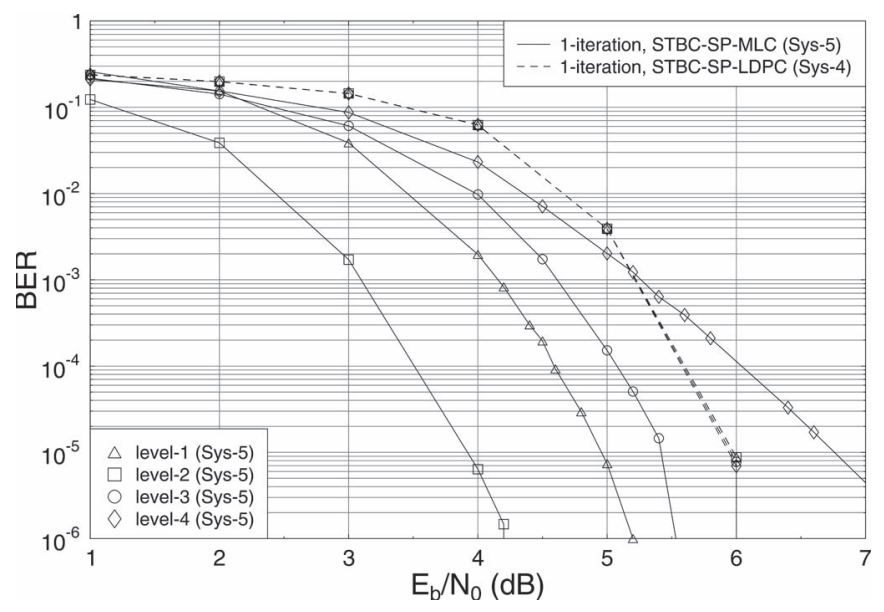

Fig. 7. BER versus $E_{b} / N_{o}$ performance of the UEP1 mapping scheme (System-5) while retaining the coding rate of L1-BSA mapping.

component code in the MLC structure. This will reduce the associated error floor. The dotted line shown in Fig. 6 indicates the BER performance of System-1 with each of its LDPC component codes having ten times the original block length of Table IV after four iterations.

We next investigate the attainable performance of the UEP scheme detailed in Section V. Fig. 7 shows the effect of invoking the UEP1 mapping scheme (System-5), where the bits mapped to level 2 of the SP constellation are protected more strongly than the other protection levels, exhibiting an $E_{b} / N_{0}$ gain of around $1 \mathrm{~dB}$ over the other classes after a single iteration and around $2 \mathrm{~dB}$ gain compared with all the protection levels shown in System-4. This performance comparison is carried out using only one outer iteration for a typical low-latency delaysensitive multimedia application that requires UEP capability. Our CF optimized using the BSA may also be used for possible extension of the UEP classes to even larger M-D SP constellations in multimedia communications. By contrast, all the bits in a single-class STBC-SP-LDPC $(2560,1280)$ System-4 exhibit a similar BER performance.

\section{CONCLUSION}

In this paper, we proposed a novel STBC-SP-MLC turbo transceiver that exploits both the space diversity of STBCs and the joint-SP symbol design of the two time slots. The proposed schemes benefit from a substantial diversity gain and from the employment of SP constellations using different mapping strategies. A novel equivalent capacity-based code rate design was invoked to calculate the coding rate of each MLC component code. It is worth mentioning that by invoking an M-D SP demapper, we can achieve a coding advantage of around $3 \mathrm{~dB}$ using $I=4$ iterations compared with conventional QPSK-based UP, as shown in Table IV. Furthermore, we designed a number of new mapping schemes, which span across different SP layers for $M=16$, while minimizing the $\mathrm{CF}$ of (11) using the BSA. A novel UEP design was also proposed, which used a modified CF optimized by the BSA.

\section{ACKNOWLEDGMENT}

The authors would like to thank M. El-Hajjar for the useful discussion that polished up the fine detail of this paper and the anonymous reviewers for their valuable comments that led to a substantially improved manuscript.

\section{REFERENCES}

[1] H. Imai and S. Hirawaki, "A new multilevel coding method using error correcting codes," IEEE Trans. Inf. Theory, vol. IT-23, no. 3, pp. 371377, May 1977.

[2] S. M. Alamouti, "A simple transmit diversity technique for wireless communications," IEEE J. Sel. Areas Commun., vol. 16, no. 8, pp. 1451-1458, Oct. 1998.

[3] L. H.-J. Lampe, R. Schober, and R. F. H. Fischer, "Multilevel coding for multiple-antenna transmission," IEEE Trans. Wireless Commun., vol. 3, no. 1, pp. 203-208, Jan. 2004.

[4] D.-F. Yuan, F. Zhang, A.-F. So, and Z.-W. Li, "Concatenation of space-time block codes and multilevel coding over Rayleigh fading channels," in Proc. IEEE Veh. Technol. Conf-_Fall, Atlantic City, NJ, Oct. 2001, pp. 192-196.

[5] P. A. Martin, D. M. Rankin, and D. P. Taylor, "Multi-dimensional space-time multilevel codes," IEEE Trans. Wireless Commun., vol. 5, no. 11, pp. 3287-3295, Nov. 2006.

[6] V. Tarokh, H. Jafarkhani, and A. R. Calderbank, "Space-time block codes from orthogonal designs," IEEE Trans. Inf. Theory, vol. 45, no. 5, pp. 1456-1467, Jul. 1999.

[7] W. Su, Z. Safar, and K. J. R. Liu, "Space-time signal design for timecorrelated Rayleigh fading channels," in Proc. IEEE Int. Conf. Commun., Anchorage, AK, May 2003, pp. 3175-3179.

[8] O. Alamri, B. L. Yeap, and L. Hanzo, "Turbo detection of channel-coded space-time signals using sphere packing modulation," in Proc. IEEE Veh. Technol. Conf.-Fall, Los Angeles, CA, Sep. 2004, pp. 2498-2502.

[9] O. Alamri, B. L. Yeap, and L. Hanzo, "A turbo detection and spherepacking-modulation-aided space-time coding scheme," IEEE Trans. Veh. Technol., vol. 56, no. 2, pp. 575-582, Mar. 2007.

[10] L. Hanzo, T. H. Liew, and B. L. Yeap, Turbo Coding, Turbo Equalisation and Space Time Coding for Transmission Over Wireless Channels. New York: Wiley-IEEE, 2002.

[11] F. Shreckenbach, N. Görtz, J. Hagenauer, and G. Bauch, "Optimization of symbol mappings for bit-interleaved coded modulation with iterative decoding," IEEE Commun. Lett., vol. 7, no. 12, pp. 593-595, Dec. 2003.

[12] J. H. Conway and N. J. Sloane, Sphere Packings, Lattices and Groups. New York: Spinger-Verlag, 1999.

[13] U. Wachsmann, R. F. H. Fischer, and J. B. Huber, "Multilevel codes: Theoretical concepts and practical design rules," IEEE Trans. Inf. Theory, vol. 45, no. 5, pp. 1361-1391, Jul. 1999.

[14] R. Gallager, Information Theory and Reliable Communication. New York: Willey, 1968.

[15] R. Gallager, Low Density Parity Check Codes. Cambridge, MA: MIT Press, 1963. 
[16] R. Y. S. Tee, O. Alamri, and L. Hanzo, "Joint design of twin-antenna assisted space-time multilevel sphere packing aided coded modulation," in Proc. IEEE VTC - Fall, Montreal, QC, Canada, Sep. 2006, pp. 1-5.

[17] S. X. Ng and L. Hanzo, "On the MIMO channel capacity of multidimensional signal sets," IEEE Trans. Veh. Technol., vol. 55, no. 2, pp. 528-536, Mar. 2006

[18] L. He and H. Ge, "A new full-rate full-diversity orthogonal space-time block coding scheme," IEEE Commun. Lett., vol. 7, no. 12, pp. 590-592, Dec. 2003.

[19] M. Isaka, R. H. M Zaragoza, M. P. C. Fossorier, S. Lin, and H. Imai, "Multilevel coded 16-QAM modulation with multistage decoding and unequal error protection," in Proc. Global Commun. Conf., Sydney, Australia, Nov. 1998, vol. 6, pp. 3548-3553.

[20] G. Ungerböck, "Channel coding with multilevel/phase signals," IEEE Trans. Inf. Theory, vol. IT-28, no. 1, pp. 55-67, Jan. 1982.

[21] F. Guo, "Low density parity check coding," Ph.D. dissertation, Univ. Southampton, Southampton, U.K., 2005.

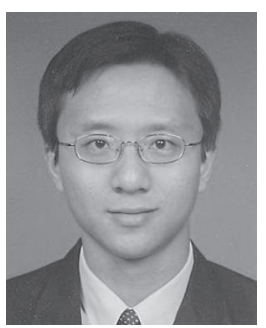

Ronald Yee Siong Tee received the B.Eng. degree (with first-class honors) in electrical and electronics engineering from the University of Manchester Institute of Science and Technology, Manchester, U.K., in 1999 and the M.Sc. degree in electrical engineering from the National University of Singapore, Singapore, in 2004. He is currently working toward the Ph.D. degree with the Communications Group, School of Electronics and Computer Science, University of Southampton, Southampton, U.K.

From 2001 to 2003, he was with Nortel Networks Switzerland and a local Singapore IT company, where he worked in data and optical networks. His research interests include iterative decoding, sphere packing modulation, and coded modulation schemes.

Dr. Tee was the recipient of several academic awards, including the Overseas Research Scheme, the ASEAN scholarship, and Malaysian Government studentships.

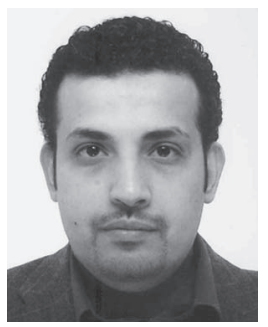

Osamah Rashed Alamri received the B.S. degree (with first-class honors) in electrical engineering from King Fahd University of Petroleum and Minerals, Dhahran, Saudi Arabia, in 1997, where he was ranked first with a 4.0 GPA, the M.S. degree in electrical engineering from Stanford University, Stanford, CA, in 2002, and the Ph.D. degree from the University of Southampton, Southampton, U.K., in 2007.

He is currently with the School of Electronics and Computer Science, University of Southampton. He has published in excess of 20 research papers. His research interests include sphere packing modulation, space-time coding, turbo coding and detection, multidimensional mapping, and MIMO systems.

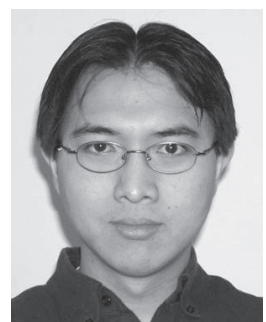

Soon Xin Ng received the B.Eng. degree (first class) in electronics engineering and the Ph.D. degree in wireless communications from the University of Southampton, Southampton, U.K., in 1999 and 2002, respectively.

From 2003 to 2006, he was a Postdoctoral Research Fellow, working on collaborative European research projects known as SCOUT, NEWCOM, and PHOENIX. Since August 2006, he has been a Lecturer in wireless communications with the University of Southampton. He has published numerous papers and coauthored a book. His research interests are mainly in adaptive coded modulation, channel coding, space-time coding, joint source and channel coding, OFDM, and MIMO.

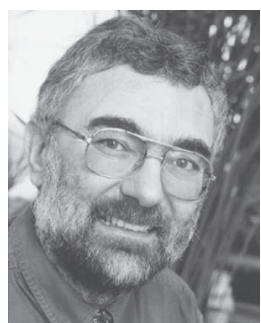

Lajos Hanzo (F'04) received the First-Class degree in electronics and the Doctorate degree from the Technical University of Budapest, Budapest, Hungary, in 1976 and 1983, respectively, and the Doctor of Sciences (D.Sc.) degree from the University of Southampton, Southampton, U.K., in 2004.

During his career in telecommunications, he has held various research and academic posts in Hungary, Germany, and the U.K. Since 1986, he has been with the School of Electronics and Computer Science, University of Southampton, where he holds the Chair in telecommunications. He has coauthored 15 books, totaling 10000 pages, on mobile radio communications, published in excess of 750 research papers, is acting as Editor-in-Chief of the IEEE Press, has acted as TPC Chair of numerous major Institution of Electrical Engineers and IEEE conferences, presented various keynote lectures, and has been awarded a number of distinctions. He currently heads an academic research team, working on a range of research projects in the field of wireless multimedia communications sponsored by industry, the Engineering and Physical Sciences Research Council (EPSRC) U.K., the European IST Programme, and the Mobile Virtual Centre of Excellence (VCE), U.K. He is an enthusiastic supporter of industrial and academic liaison. He offers a range of industrial courses.

Dr. Hanzo is an IEEE Distinguished Lecturer of both the Communications and the Vehicular Technology Societies, and a Fellow of the Institution of Electrical Engineers and the Royal Academy of Engineering. He is an Editorial Board Member of the PROCEEDINGS OF THE IEEE and a Governor of the IEEE VT Society. 